\title{
Norois
}

Environnement, aménagement, société

$228 \mid 2013$

La patrimonialisation des paysages de l'eau dans l'ouest de la France

\section{Les ambiguiités et les limites de la construction patrimoniale d'une industrie en activité. Le cas de la Centrale thermique de Cordemais}

Ambiguities and boundaries of the heritage production of a manufactory in activity. The case study of the coal plant of Cordemais

\section{Clément Colin, Nacima Baron-Yelles et Jean-Louis Kerouanton}

\section{(2) OpenEdition}

Journals

Édition électronique

URL : http://journals.openedition.org/norois/4736

DOI : $10.4000 /$ norois.4736

ISBN : 978-2-7535-2918-2

ISSN : 1760-8546

Éditeur

Presses universitaires de Rennes

Édition imprimée

Date de publication : 30 septembre 2013

Pagination : 65-75

ISBN : 978-2-7535-2883-3

ISSN : 0029-182X

Référence électronique

Clément Colin, Nacima Baron-Yelles et Jean-Louis Kerouanton, « Les ambiguïtés et les limites de la construction patrimoniale d'une industrie en activité. Le cas de la Centrale thermique de Cordemais », Norois [En ligne], 228 | 2013, mis en ligne le 30 septembre 2015, consulté le 19 avril 2019. URL : http:// journals.openedition.org/norois/4736 ; DOI : 10.4000/norois.4736 


\title{
Les ambiguïtés et les limites de la construction patrimoniale d'une industrie en activité Le cas de la centrale thermique de Cordemais
}

\author{
Ambiguities and Boundaries of the Heritage Making of an Industry in Activity. The Case Study of the \\ Coal Plant of Cordemais
}

\section{Clément Colin ${ }^{* a}$ Nacima Baron-Yelles $^{\mathrm{b}}$ Jean-Louis Kerouanton ${ }^{\mathrm{c}}$}

\footnotetext{
* Auteur correspondant

a LVMT - UMR INRETS T9404, (École des Ponts ParisTech), 6-8 avenue Blaise-Pascal, Champs-sur-Marne -

77455 MaRnE-LA-VALLÉE Cedex 2 (colinclement@orange.fr)

b LVMT - UMR INRETS T9404, (Université Paris Est Marne-la-Vallée)

6-8 avenue Blaise-Pascal, Champs-sur-Marne - 77455 MARne LA VAlLÉE Cedex 2 (nacima. baron-yelles@laposte.net)

${ }^{c}$ Centre François Viète - EA 1161, (Université de Nantes), Faculté des Sciences et des Techniques, 2 rue de la

Houssinière, BP 92208 - 44322 NANTES Cedex 3 (jean-louis.kerouanton@univ-nantes.fr)
}

Résumé : À partir de l'exemple de la centrale thermique de Cordemais, l'article interroge les limites de la construction patrimoniale et de son extension aux industries en activité. Sortant du référentiel patrimonial traditionnel par son usage, cet équipement producteur d'électricité tend pourtant à être interrogé dans ses dimensions patrimoniales. À partir d'entretiens semi-directifs auprès d'acteurs territoriaux de l'estuaire de la Loire, nous examinons les chainons explicatifs de cette désignation patrimoniale et de ses limites.

\begin{abstract}
With the example of the Cordemais coal plant, the article deals with the limits of the extension of the heritage making to industrial activities. The heritage dimension of an energy plant is questioned despite its purpose takes it out of the traditional heritage framework. Through the analysis of semi-structured interviews with local stakeholders of the Loire estuary, we examine explanatory factors of this heritage nomination. Moreover, the notion of heritage, its evolution and its boundaries are questioned by case study.
\end{abstract}

Mots clés : patrimonialisation - territorialisation - centrale thermique - estuaire

Keywords: heritage making - territory making - coal plant - estuary

Amplement analysé ou dénoncé par de nombreux auteurs, le phénomène patrimonial touche aujourd'hui toutes les formes de bâtis ou de lieux pour leurs caractéristiques propres et pour ce qu'ils représentent pour la société. Défini de manière très générale par Françoise Choay (1992) comme « une part d'un héritage qui augmente par annexion de nouveaux types de biens et par élargissement du cadre chronologique et des aires géographiques à l'intérieur desquels ces biens s'inscrivent », il reste une notion relativement floue et difficilement délimitable dans le temps et l'espace. Longtemps objet 
des historiens et historiens de l'art (Riegl, 1903; Choay, 1992; Lamy, 1993; Poulot [dir.], 1998), le patrimoine est, depuis les années 1980-1990, étudié par les autres disciplines de sciences sociales comme la géographie (Di Méo, 1994, 1998; Veschambre et Gravari-Barbas, 2003; Gravari-Barbas [dir.], 2005; Veschambre, 2007, 2008) ou encore la sociologie (Bourdin, 1984; Davallon, 2006 ; Jeudy, 2008; Amougou, 2011) sous l'angle de sa construction. Face à son extension à divers biens, les services des inventaires et les scientifiques le répartissent en différentes catégories plus ou moins bien distinctes : nous parlons ainsi de patrimoine urbain, historique, industriel, rural, naturel, mémoriel, culturel et immatériel. Face à ce constat, Pierre Henri Jeudy (2008) critique la surabondance des lieux de mémoire qu'il qualifie de "garantie contre l'oubli ». D'autres, à l'instar de Vincent Veschambre (2008), parlent de droit à l'accès au « conservatoire de l'espace ». Le patrimoine est passé en un siècle d'un donné historique à un construit social et d'une conception a-territoriale à une dimension territoriale. Guy Di Méo (1994) note la parenté conceptuelle entre territoire et patrimoine. Le géographe met en évidence l'étroite relation des fonctions patrimoniales et territoriales dans la formation du lien spatial des individus ou de groupes d'individus.

À partir de la crise des années 1980 et l'apparition de vastes friches en plein cœur des villes, les industries font leur entrée dans le monde du patrimoine. Le concept de «patrimoine industriel » est issu de l'« archéologie industrielle ${ }^{1}$ », née au Royaume-Uni dans les années 1950 (Edelblutte, 2008). Il associe « la collecte des sources écrites, familières à l'histoire, et à la pratique assidue du terrain » (Andrieux, 1992). La plupart des travaux sur les héritages industriels urbains ou ruraux portent avant tout sur des établissements délaissés ou des documents d'archives. Les équipements encore en activité ne sont pas considérés comme du patrimoine. La visite d'entreprise et le tourisme industriel tendent, cependant, à mettre à mal cette conception. Phénomène relativement récent ${ }^{2}$, il reste difficile à évaluer et à définir (Morice, 2006). André Marcon, Pierre

1. L'archéologie industrielle peut être définie comme «l'activité scientifique qui se donne pour objet d'éclairer un corpus cohérent d'éléments rassemblés sous le vocable de patrimoine industriel » (Andrieux, 1992).

2. Chaque année, en France, environ 1400 entreprises, musées du patrimoine ou sites industriels ont ouverts leurs portes pour accueillir $20 \mathrm{mil}$ lions de visiteurs (Bianchini, 2008).
Preuilh et Stéphanie Ksouri (2000) distinguent le tourisme de la visite d'entreprises en activité, le tourisme scientifique et le tourisme de patrimoine industriel $^{3}$. Il apparait clairement que les visiteurs s'intéressent à l'activité même de l'objet industriel.

Avec l'évolution du tourisme industrielle et technique et de la visite d'entreprises, nous faisons l'hypothèse d'une nouvelle extension de la définition patrimoniale qui peut désigner aujourd'hui des objets dont l'usage est encore d'actualité. À partir de l'exemple de la centrale thermique de Cordemais, nous interrogeons les limites de la construction patrimoniale et de son extension aux équipements industriels en activité. En fonction depuis 1970, elle est désignée aujourd'hui par la municipalité de Cordemais et par de nombreux acteurs territoriaux comme un élément constitutif, "patrimonial » et «identifiant » de la commune et de l'estuaire de la Loire où elle se situe. L'article se base sur une enquête effectuée à partir de dix entretiens semidirectifs avec ${ }^{4}$ : la responsable de la communication de l'unité de production EDF de Cordemais, le maire de Cordemais, la directrice de l'association Estuarium ${ }^{5}$, la directrice de l'Office du tourisme Cœur d'estuaire, un élu régional Europe Ecologie-les verts, une chargée de communication de la biennale « Estuaire », la directrice du Conservatoire régional des rives de Loire et de ses affluents ${ }^{6}$, le chargé du label du " patrimoine $\mathrm{xx}^{\mathrm{e}}$ siècle ${ }^{7}$ » à la DRAC Pays de la Loire, le directeur du Groupement d'intérêt général (GIP) Loire estuaire 8 et le chargé de communication du syndicat intercommunal de chasse au gibier d'eau de la basse Loire nord ${ }^{9}$ (SICGEBLN).

3. Relatif « à des sites, des productions et des savoir-faire qui font partie de l'histoire industrielle nationale ou locale » (Marcon et al., 2000).

4. Nous choisissons de conserver l'anonymat de ces individus. La méthode employée est celle de la sociologie compréhensive : donner et tirer du sens aux discours recueillis restent le principal objectif.

5. Fondée en 1994 dans le cadre de l'élaboration de la Directive Territoriale d'Aménagement de l'estuaire de la Loire et rattachée au service régional des inventaires, sa mission est d'identifier des éléments perçus comme pouvant être patrimoniaux et « identitaires » pour ce territoire.

6. Structure associative créée en 1992 à l'initiative du Conseil Régional des Pays de la Loire et les Conseils Généraux de Maine et Loire et de Loire Atlantique, elle a pour mission statuaire la préservation et la valorisation des paysages et de la biodiversité de la Loire et de ses Affluents en région Pays de la Loire.

7. Ce label est mis en place par les circulaires du 18 juin 1999 et celle du $1^{\text {er }}$ mars 2001, issus de la Recommandation Européenne "relatives à la protection du patrimoine architectural du vingtième siècle » adoptée le 9 septembre 1991.

8. Cette structure nommée GIP Loire Estuaire depuis 2004, a pour objectif de capitaliser toutes les connaissances et créer des indicateurs sur le long terme. Elle porte des programmes de restauration de l'estuaire.

9. Association fondée en 1959, son objectif est de gérer la chasse au gibier d'eau (canard...). Neuf communes de la rive nord de l'Estuaire se sont 
À partir de ces discours réalisés en 2010 et 2011 , nous articulons notre propos autour de deux questionnements :

- Pourquoi et comment la centrale est-elle reconnue et acceptée comme élément caractéristique du territoire estuarien par les différents acteurs territoriaux rencontrés?

- Peut-on réellement utiliser le terme de «patrimoine » pour désigner la centrale thermique de Cordemais?

Après avoir présenté brièvement la centrale thermique à flammes de Cordemais, nous nous intéressons aux processus qui conduisent à sa désignation comme élément identificateur du territoire estuarien par les acteurs territoriaux rencontrés. Nous préférons les termes «identifiant » ou «identification » à celui d'«identité » ou d'« identitaire » afin de privilégier l'analyse du processus et des stratégies et des acteurs liés plutôt que de nous intéresser à l'identité qui est de plus une notion complexe renvoyant à diverses approches et théories souvent contradictoires (Brubaker, 2001). Dans le même sens, nous préférons analyser les processus de patrimonialisation plutôt que l'objet patrimoine. Enfin, dans un troisième temps, nous allons nous intéresser aux ambiguïtés de cette reconnaissance.

\section{Présentation de la centrale THERMIQUE À FLAMMES de Cordemais}

Dans les années 1960, les sites accessibles par voie d'eau sont privilégiés par EDF pour implanter de nouvelles centrales thermiques à flammes. L'État considère que la consommation électrique double tous les dix ans passant de 54 milliards de kWh en 1956 à 109 milliards en 1966 (Kerouanton et Lemaître, 1996, p. 12). Pour lui, il est nécessaire de construire de nouvelles unités de production plus puissantes. Du constat d'un déficit énergétique dans l'ouest de la France, EDF cherche à équiper en énergie la Bretagne et ses alentours dont la population est hostile au nucléaire. Les deux premiers groupes de production de $600 \mathrm{MW}$ sont celui de Porcheville en 1968, du Havre en 1969 et le troisième est celui de Cordemais.

regroupées pour mettre leur territoire de chasse en commun et pour proposer un territoire de chasse beaucoup plus grand et mieux gérer aux adhérents.
À l'échelle de l'estuaire de la Loire, la centrale thermique s'inscrit dans la continuité de l'histoire industrielle de ce territoire. Trois centrales électriques se situaient dans Nantes et le Bas Chantenay entre 1891 et 1912 (Guillaume, 2009). En 1953, EDF choisit Cheviré pour implanter une centrale thermique. Celle-ci ferme en 1986 car elle est moins performante que la centrale de Cordemais, en fonctionnement depuis 1970. Elle permet aujourd'hui de fournir de l'électricité à 2,5 millions d'habitants. Tandis qu'elle devait brûler entièrement du fioul, la crise de 1974 entraîne le passage des tranches suivantes au charbon (Halgand et Guillaume, 2007). La centrale est située sur un seul site : les anciennes îles de la Calotte et de la Nation réunies au XIX ${ }^{\mathrm{e}}$ siècle. Les terrains étaient des pâturages inondables qu'il a fallu remblayer avant la construction de la centrale. Celle-ci se compose depuis 2010 de quatre tranches - deux qui fonctionnent au fioul et deux qui fonctionnent au charbon -, d'un parc à charbon, de sept dépôts de fioul et d'un dépôt d'eau. L'architecture principale a été conçue par Michel Homberg, l'architecte de la centrale électrique de Cheviré. L'une de ces caractéristiques majeures sont les cheminées qui s'élèvent dans le ciel jusqu'à 220 mètres. Cela les rend visibles du pont de Cheviré, à la sortie de Nantes, au pont de Saint-Nazaire, à l'embouchure de l'estuaire. La centrale se situant au cœur des marais estuariens, au paysage plat et monotone, elles sont d'autant plus voyante. Aujourd'hui, il n'en reste plus que trois ${ }^{10}$ : une de 150 mètres de haut pour les tranches 2-3, une de 220 mètres pour les tranches 4-5 et une de 220 mètres construite en 1996 pour la dépollution des fumées (Kerouanton et Lemaître, 1996) (figure 1).

\section{LE PROCESSUS DE RECONNAISSANCE ET DE DÉSIGNATION DE LA CENTRALE THERMIQUE COMME ÉLÉMENT IDENTIFIANT LE TERRITOIRE ESTUARIEN}

La centrale thermique est construite en élément « identifiant » d'abord à l'échelle de la commune puis à celle de l'estuaire de la Loire. Nous tentons de comprendre les processus qui permettent cette désignation.

10. La première liée à la tranche 1 en inactivité depuis 1996 a été déconstruite. 


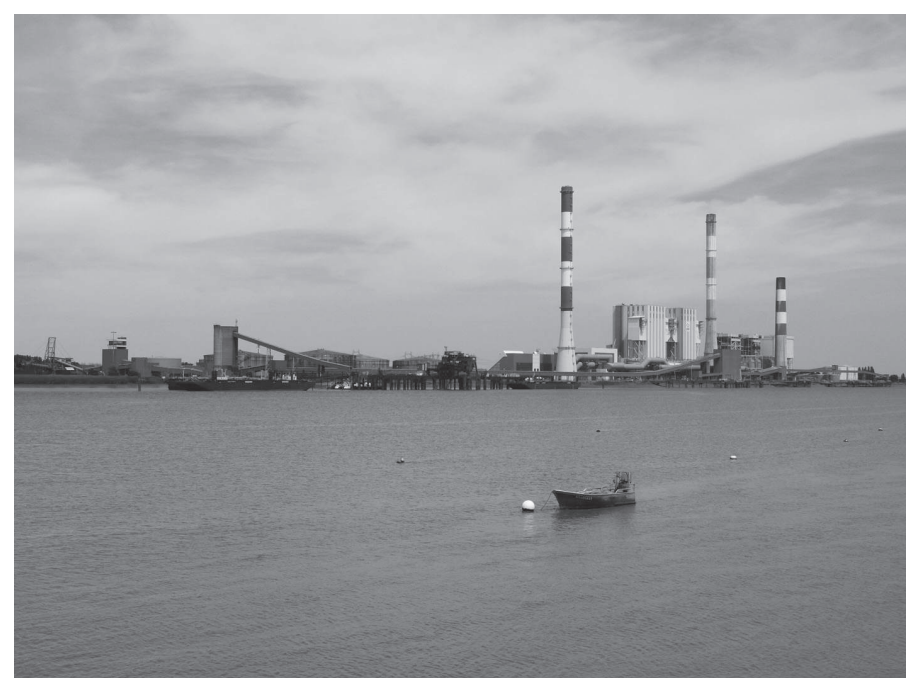

Figure 1 : La centrale de Cordemais vue de l'autre rive de l'estuaire de la Loire (Photo de Clément Colin, juin 2010)

View of Cordemais coal plant from the other side of the Loire estuary

\section{À l'échelle communale : de la rupture à l'ancrage territorial}

Symbole de la modernité, la centrale thermique amène emploi, attractivité et revenu pour la commune de Cordemais par le biais des taxes professionnelles. Elle lui permet d'avoir des ressources financières qui l'aident à implanter des équipements municipaux dignes d'une ville moyenne. Au vu des statistiques de l'INSEE, la centrale thermique a un impact sur la croissance démographique sur la commune lors de son implantation. Entre 1968 et 1975, il y a eu en moyenne une augmentation de la population de 3,4\% par an dans la commune, contrairement à Saint-Etienne-de-Montluc, commune voisine, qui connaît une augmentation de $1,2 \%^{11}$ sur la même période. Si la centrale a, selon le maire de Cordemais, une influence quelque peu négative sur la commune en termes d'image, il n'y a ni baisse ni stagnation du nombre d'habitants depuis les années 1960. Elle marque l'organisation socio-économique de la commune qui, avant 1970, était essentiellement rurale.

11. Insee, RP1968 à 1990 dénombrements - RP1999 et RP2008 exploitations principales - État civil.

\section{Le maintien du petit port comme déclen- cheur du changement d'attitude d'EDF vis-à-vis du territoire}

Le changement de regard sur la centrale thermique de Cordemais de la part de ses dirigeants mais aussi de la population et des élus de la commune est, tout d'abord, dû au fait de son activité qui permet le maintien du petit port de Cordemais. Ces « petits ports ${ }^{12}$ » occupent une place importante dans le fonctionnement de l'espace estuarien du Moyen-âge au $\mathrm{Xx}^{\mathrm{e}}$ siècle. Ces ports d'étiers servaient d'abri aux navires de l'estuaire (Lemaître et Lemerle, 2004) mais, à partir de la fin du XIX siècle, les différents travaux d'endiguements et de canalisation de l'estuaire de la Loire accentuent les problèmes d'envasement endémiques. Condamnées à l'abandon et à l'oubli dans le reste de l'estuaire, les traces de ces activités portuaires sont aujourd'hui perçues par les élus et les habitants comme des témoins des points de contacts perdus avec le fleuve. Les communes tentent de les faire vivre malgré le problème récurrent d'envasement. L'activité portuaire de Cordemais, très dynamique jusqu'au $\mathrm{xx}^{\mathrm{e}}$ siècle, devait connaître la même trajectoire que celle des communes voisines. L'association des Usagers et Amis du Port de Cordemais avait à l'origine pour ambition de préserver le port de Cordemais contre les méfaits possibles de la centrale ${ }^{13}$. À l'inverse, par le rejet d'eau dans l'étier de l'estuaire où le port est présent, l'activité industrielle repousse la vase accumulée et rend l'arrivée de bateaux possible. La directrice de l'association Estuarium ${ }^{14}$ évoque cette prise de conscience : "Il y a une chose intéressante c'est qu'on a commencé à faire prendre conscience aux dirigeants de la centrale que cette centrale avait tout son intérêt sur Cordemais, et pas que d'un point de vue financier parce qu'elle avait sawvé un des derniers petits ports encore utilisés aujourd'hui qui est le petit port de Cordemais. » Les recherches menées sur les petits ports de l'estuaire en 2004 et 2005, par Yves Lemaître et Eric Lemerle, dans le cadre de l'asso-

12. Nom donné par les ingénieurs des Ponts et Chaussées au xixe aux ports situés entre Nantes et Saint-Nazaire.

13. Guy Letellier, président de l'association Usagers et amis du port de Cordemais depuis 2002, interrogé par Béatriz Cort, Estuarium, disponible sur [http://petits.ports.free.fr/letellier2.pdf].

14. Fondée en 1994 dans le cadre de l'élaboration de la Directive territoriale d'aménagement (DTA) de l'estuaire de la Loire et rattachée au service régional des inventaires (autrefois à la DRAC), sa mission est d'identifier des éléments perçus comme pouvant être patrimoniaux et «identitaires » pour ce territoire. 
ciation, font prendre conscience à EDF de l'impact positif que son activité peut avoir sur le territoire. Le maintien d'un patrimoine rend légitime la présence de la centrale thermique. La plupart des personnes interrogées évoquent le maintien du patrimoine fluvial de Cordemais comme un facteur d'ancrage d'EDF sur la commune. Elle permet de faire perdurer un lien vivant avec le passé et l'histoire de l'estuaire.

\section{EDF à la recherche d'ancrage territoriale}

EDF cherche aujourd'hui à s'ancrer davantage sur le territoire, notamment en aidant financièrement les autres activités traditionnelles. Le maire de Cordemais évoque le fait qu'EDF participe avec la commune et l'Etat à la remise en fonctionnement d'une écluse des marais détruite par la tempête Xynthia en $2010^{15}$ : «Il y a une vanne-écluse qui évite que l'eau entre dans le marais qui a été détruite par la tempête Xynthia, bon on a trouvé de l'argent au niveau de l'Etat et tout ça mais la centrale de Cordemais et la mairie de Cordemais qui n'ont rien a voir la dedans on va donner une subvention chacun. Donc on a quand même des relations assez fortes. » Plus qu'une simple ressource financière par la taxe professionnelle, EDF veut se transformer en acteur du territoire pour améliorer son image. La responsable de la communication de l'unité de production de Cordemais affirme cette ambition lors de notre rencontre : "Nous voulons être un acteur incontournable $d u$ territoire. » L'estuaire est perçu par une majorité d'acteurs territoriaux rencontrés comme un espace surprotégé par des règlementations pour la protection de la biodiversité. Les industriels cherchent à mieux s'intégrer à ce cadre. Cela ne se fait pas sans difficultés. En complément à ces stratégies, EDF a l'avantage, contrairement à Total (avec la raffinerie de Donges) de n'avoir pas connu, de problèmes de pollution «visibles » ou polémiques et encore avoir eu besoin d'engager une lutte contre les écologistes de l'estuaire ${ }^{16}$. La centrale thermique n'a pas détruit des activités déjà existantes sur son lieu d'implantation. Pour le maire de Cordemais, l'activité de la centrale thermique ne dérange pas le quotidien des

15. Cette information est vérifiable sur le site internet du GIP Loire Estuaire (information vérifiée le 18 janvier 2012).

16. Nous faisons référence ici au combat écologiste contre le projet d'extension portuaire de Donges est qui s'est terminé en 2009 par leur victoire et l'abandon du projet voulu par l'autorité portuaire. habitants : «Les gens l'ont accepté : après tout c'est là, ça pollue pas tant que ça, ça fait pas de nuisance particulière. On voit qu'autour il y a des arbres, il y a des gens qui vivent et ils n'ont pas l'air d'être malade. " EDF montre qu'elle prend en compte l'environnement à travers des certifications européennes ou nationales de «bonne conduite écologique».

L'entreprise ouvre désormais la centrale thermique au public local à travers un espace dédié à l'information sur son fonctionnement. Elle fait aussi partie d'une association valorisant les ouvertures d'entreprises et le tourisme industriel en région Pays de la Loire : "Visitez nos entreprises en région Pays de la Loire ${ }^{17}$. » À travers cette structure associative, la centrale est montrée comme un site visitable par les touristes intéressés. Dans les faits, elle est très peu visitée, essentiellement par un public venant de Cordemais, des communes voisines et par des scolaires. Perçue comme une politique d'entreprise, la recherche d'ancrage d'EDF sur le territoire parait légitime face à la pression conservatrice du milieu estuarien. Le point de vue du responsable de la communication du SCGEBLN va dans ce sens : "C'est de la politique d'entreprise. Tout le monde veut développer du développement durable et ça c'est aussi un peu pour se donner bonne conscience. " Le directeur du GIP Loire estuaire donne, quant à lui, raison aux industriels de mener telles stratégies de communication : "Est ce que les industries sont dans une logique de valorisation du territoire estuarien? En tout cas elles en profitent et elles ont raison de le faire. » Dans ce cadre règlementaire de protection de la nature, la place de l'industrie dans l'estuaire est questionnée (Guillaume, 2009) que ce soit pour son activité, la génération d'emplois directs ou encore son adaptation à l'environnement concurrentiel international. Les industriels ont tout intérêt à mieux se lier aux évolutions de leur territoire d'implantation. Seul équipement électrique dans le secteur, la centrale est une opportunité économique et strétégique atout pour EDF qui veut maintenir sa position dans la région.

17. À la suite d'une étude faite par l'Observatoire régional du tourisme en 1999, le comité régional du tourisme, la chambre régionale de commerce et d'industrie, les CCI locales et 24 entreprises créent en septembre 2001 cette association. Elle a l'appuie de l'Etat et de la région dans le cadre du contrat État-région depuis le 7 mars 2002 (Charuel, Leblanc, Brin, 2006). 


\section{La stratégie de communication de la municipalité de Cordemais}

Aujourd'hui, avec l'arrivée de nouveaux habitants ${ }^{18}$, la municipalité de Cordemais, veut tirer avantage de la présence de cette centrale thermique qui intrigue les habitants et les quelques visiteurs par son gigantisme. Le maire de Cordemais évoque un changement de stratégie par rapport à son prédécesseur : "Le maire avant avait une vision différente de la mienne. Il avait la nostalgie d'avant la centrale.» Il mène une politique de communication en montrant la centrale EDF comme élément incontournable du territoire faisant partie de "l'identité de la commune». Il explique sa démarche : "Quand on dit infrastructure industrielle, on dit c'est par nature mawvais. On prend l'action dans l'autre sens en disant cette équipement il est énorme mais il a des formes, une silhouette assez élancée avec ses cheminées, il a quelque chose. Donc on dit que c'est une identité forte de Cordemais et cette construction elle est là on doit la valoriser et elle doit valoriser le territoire. » Cette stratégie pour sensibiliser la population habitante et les nouveaux arrivants est basée sur le site Internet de la commune mais aussi sur le Mag Info de Cordemais (le mensuel de la commune). Les Journées Européennes du Patrimoine sont aussi l'occasion pour l'ouvrir et la faire visiter. En partenariat entre EDF, la commune de Cordemais, la communauté de communes Cœur d'Estuaire et l'Office du Tourisme Cœur d'Estuaire, la visite animée de la centrale accueille de nombreuses personnes.

À l'échelle communale nous assistons à des processus de légitimation de la présence et de l'activité de la centrale née de la volonté conjointe d'EDF et du maire. La pérennisation du port de Cordemais ainsi que la méconnaissance ou l'inexistence relative de problèmes environnementaux majeurs liés à l'activité de la centrale thermique font que celle-ci tend à être acceptée et reconnue au niveau local comme élément faisant sens dans ce territoire.

18. Sur la période 1990-1999, la variation annuelle moyenne de la population était de 0,7\% et de 1999-2008 elle était de 1,4\% et cela essentiellement grâce à l'arrivée de nouveaux habitants (sources : Insee, RP1968 à 1990 dénombrements - RP1999 et RP2008 exploitations principales - État civil).

\section{À l'échelle métropolitaine : un repère et un symbole dans le paysage estuarien}

Protégé et préservé pour sa biodiversité et son paysage, l'estuaire de la Loire est, depuis les années 90, interrogé dans ses dimensions culturelles et «identitaires ». Espace délaissé et oublié depuis la fin de l'expansion industrielle dans les années 1980, ce cœur d'estuaire fait l'objet de stratégies de changement d'image par les acteurs constructeurs de la métropole Nantes-Saint-Nazaire.

\section{La centrale thermique considérée comme un élément du paysage estuarien}

Que ce soit avec Estuarium qui cherche à valoriser le patrimoine de cette partie inconnue de l'estuaire ou les acteurs de la métropole de Nantes-SaintNazaire qui à travers la biennale veulent « révéler » le territoire, la centrale thermique est un élément du paysage qui intrigue et qui attise la curiosité. Visible dans le paysage, elle tend à devenir son élément identificateur. L'élu régional Europe Écologieles verts des Pays de la Loire fait une comparaison intéressante entre la tour Eiffel qui identifie Paris et la Centrale de Cordemais qui est liée à l'estuaire : "Toute proportion gardée, mettez une photo de l'estuaire sans Cordemais, à part quelques experts, personne ne reconnaîtra l'Estuaire. C'est comme mettre une photo de Paris sans la Tour Eiffel. Et c'est attaché au territoire estuarien. Vous mettez la même centrale dans la vallée à côté de Grenoble, ce ne sera pas pareil. » Le directeur du GIP Loire estuaire va dans le même sens : "Sans Cordemais on ne ferait pas l'estuaire avec ses cheminées de 220 mètres de haut. C'est lié à l'histoire, à ce qu'est l'Estuaire aujourd'hui, je ne sépare pas les choses. » La notion de paysage tend à s'imposer comme nouvelle dimension patrimoniale. L'« identité paysagère » ou encore l'« intégration paysagère » sont des formules souvent utilisées par les différents interlocuteurs. Dans leurs discours, la patrimonialité du paysage tend à s'exprimer par le visuel : c'est patrimonial car on le voit dans le paysage. Le terrain très plat et monotone confère à la centrale ce rôle de repère dans l'estuaire. Cela tend même à lui donner un caractère magique et unique. Présente et visible dans le paysage, elle existe et elle est même comparée à une église qui est repérable dans le paysage et annonce la présence d'un village : 
«Elle est là elle est dans le paysage, donc on ne peut pas dire qu'elle n'existe pas. Et nous on pense que c'est une identité de Cordemais. Comme une église est l'identité d'un bourg, la centrale est une identité de Cordemais. » La dimension paysagère marquante de la centrale semble ainsi la transformer en objet mystérieux. La directrice du Conservatoire régional des rives de Loire et de ses affluents (CORELA) explique pourquoi : "Il y a quelque chose qui est magique dans le paysage. Le paysage est relativement plat et monotone. Quand vous êtes sur le fleuve vous ne voyez rien du tout. Et soudainement vous voyez cette structure. » Le maire de Cordemais évoque, quant à lui, l'imaginaire auquel elle peut renvoyer : "Quand vous êtes sur le sillon et qu'il y a un peu de brume sur le marais, la centrale ressort, vous voyez les cheminées, et ça fait comme un bateau qui flotte sur la Loire. C'est assez étonnant. C'est une oeuvre du $\mathrm{XX}$ e siècle en tout cas, ça c'est clair. C'est quelque chose qui marque son paysage ça c'est évident. On ne peut pas dire l'inverse. »

\section{L'impact de la biennale sur l'image de la centrale thermique}

Identifier ce territoire et son devenir dans l'estuaire «métropolisé » autour de deux pôles - Nantes et Saint Nazaire - est un enjeu politique important pour légitimer cette construction territoriale et politique. De leur côté, les collectivités territoriales, et notamment Nantes Métropole et la Communauté d'agglomération de la région nazairienne et de l'estuaire (CARENE), cherchent à améliorer l'image de ce territoire par la biennale "Estuaire ». Jean Blaise, directeur de la scène nationale nantaise du «Lieu Unique » et initiateur du projet, défend l’idée selon laquelle l'art est un outil politique très efficace dans la valorisation et la reconnaissance d'un territoire. Soutenu par les élus du Syndicat Mixte du SCOT de la Métropole, le projet est mis en place par le Lieu Unique, scène culturelle nationale sous la direction du même homme. "Estuaire » est pensé pour trois années : 2007, 2009 et 2011. Pour l'équipe de la biennale, tout l'enjeu est de se présenter comme transmetteur de leurs paroles et de leurs histoires à travers l'art : «Notre projet est de révéler le territoire. » Le budget de 7,5 millions d'euros à disposition de l'équipe provient en majorité de subventions publiques provenant de collectivités terri- toriales (région des Pays de la Loire, département de Loire-Atlantique, villes de Nantes et Saint-Nazaire, Nantes Métropole, communautés de communes des bords de l'estuaire) et en partie de sponsors privés comme EDF, Suez ou Total. L'équipe avec les autres acteurs du territoire veulent mettre en place des offres touristiques à partir les seize œuvres devenus pérennes. Plutôt pour un public venant des communes de l'estuaire ou de la région, l'objectif de l'équipe de la biennale montre que leur objectif est désormais de cibler l'Île de France et les capitales européennes reliées à Nantes par voie aérienne. La biennale qui devait être organisée en 2011 a été déplacée en 2012 afin de réunir les machines de l'Ile de Nantes, les châteaux de Nantes et Estuaire en un seul événement nommé « Le Voyage à Nantes » pour positionner Nantes en une ville de la culture incontournable à l'échelle européenne.

De nombreux interlocuteurs évoquent leur fascination pour les effets de la biennale qui permet de dynamiser des communes rurales abandonnées de la population et des activités économiques de services (notamment). Dans ce cadre, l'œuvre de Tatzu Nishi a un rôle de révélateur pour la centrale et permet de la faire connaître. Nommée "Villa Cheminée ", elle se présente en cheminée tronquée ressemblant à celle de la centrale avec à son sommet une maison de lotissement pouvant être habitée (figure 2).

Les œuvres de la biennale peuvent jouer un rôle dans la mise en tourisme du territoire. L'exemple de

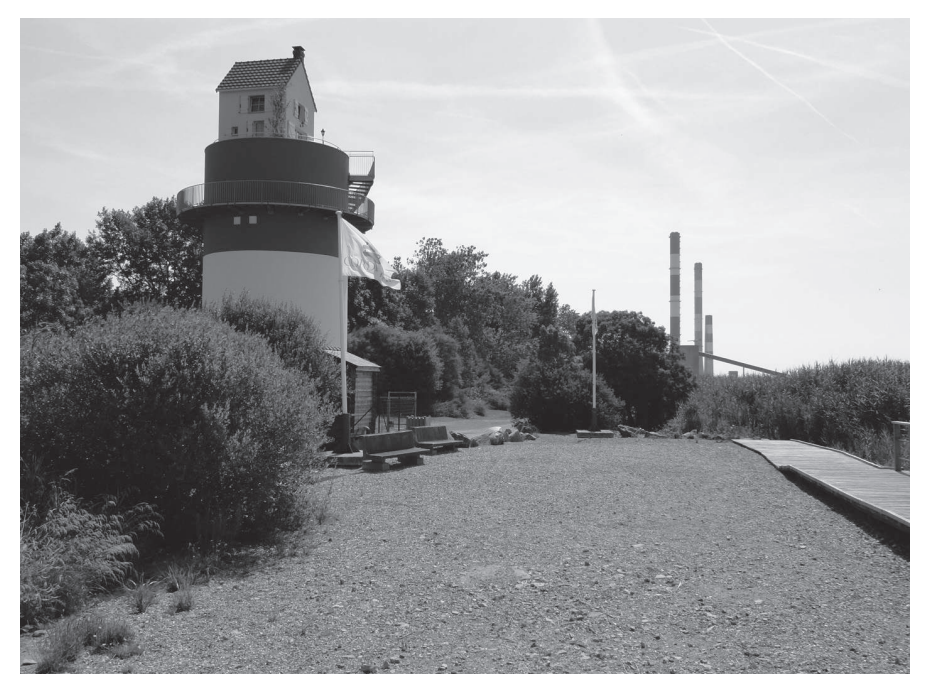

Figure 2 : L'œuvre «villa-cheminée » de Tatzu Nishi, à côté de la centrale thermique (Photo de Clément Colin, juin 2010)

The "Villa-Cheminée" of Tatzu Nischi, near of the coal plant 
l'œuvre de la villa Cheminée à côté de la Centrale de Cordemais a eu un impact sur la centrale qui est de plus en plus prise en photo et qui est considéré par l'Office du tourisme Cøur d'estuaire comme un « patrimoine insolite et industriel». L'ouverture plus régulière serait un avantage pour l'Office du tourisme Cœur d'Estuaire car il n'existe que très peu d'offres sur ce territoire. La directrice exprime ce point de vue: "Nous les deux vrais offres qu'on a aujourd'hui c'est la centrale et la villa, en site touristique. " Par ailleurs, le plan Vigipirate empêche cette possibilité de voir le jour pour le moment. À la question d'une possible ouverture durable de la centrale, la directrice de l'Office du tourisme Cœur d'estuaire montre son enthousiasme : «On aimerait bien car c'est un élément reconnu et visible pour notre territoire. »

\section{LES LIMITES DE SA DÉFINITION PATRIMONIALE}

Non reconnue ni par les acteurs traditionnels du patrimoine - DRAC et services de l'inventaire ${ }^{19}-$ ni par EDF, la centrale thermique interroge la notion de patrimoine et son extension typologique, temporelle et géographique. Cependant, peut-on réellement rapprocher les processus en cours pour la centrale thermique de Cordemais à ceux de la patrimonialisation?

Ces derniers sont tout autant ambigus que les valeurs attachées à la centrale. À la fois différents et ressemblants aux processus de patrimonialisation amplement étudiés, décrits et analysés par les sciences sociales, ce qui se déroule face à nous à propos de la centrale de Cordemais ne peut laisser indifférent un chercheur sensible à la patrimonialisation de la société. Aussi au regard des méthodes et des outils des acteurs du patrimoine où le processus de classification est dominant, la centrale pose un problème majeur de définition patrimoniale.

\section{Les raisons de la non-reconnaissance de la centrale thermique de Cordemais}

La centrale thermique de Cordemais n'est pas reconnue officiellement comme patrimoine principalement pour trois raisons. Le premier facteur pro-

19. Mise à part l'étude menée par J.-L. Kerouanton et Y. Lemaitre en 1996 sur la commune de Cordemais. vient de l'inexistence de mobilisation patrimoniale pour la protection de ce bien. Comme nous l'avons bien présenté précédemment, la centrale thermique fait l'objet de politique de valorisation, elle est mobilisée comme ressource pour le territoire. Cela s'explique tout simplement par deux faits : la centrale est encore en fonctionnement et elle est la propriété d'EDF.

Le deuxième facteur explicatif vient des propriétaires - EDF - qui ont une politique dit de «valorisation du patrimoine industriel et foncier» interne à leur service. EDF refuse le terme «patrimoine » dans son acceptation générale, considérant que cela renvoie au fait que l'activité de la centrale est terminée ou en voie de finir. Dans son dossier de presse de juillet 2009 intitulé Valorisation du patrimoine industriel et foncier, la gestion des anciens sites thermiques d'EDF, l'entreprise met en évidence que, d'ici fin 2015, l'objectif est de fermer progressivement des unités de production fonctionnant avec des centrales thermiques à flammes pour que d'autres moyens de production, plus modernes, prennent le relais. Ce qui est appelé «valorisation du patrimoine industriel et foncier » est une politique de démantèlement et de remise en état des anciens sites de centrales thermiques soit pour les réutiliser à d'autres fins, pour les moderniser avec de nouveaux moyens de production, soit les vendre ou les rendre aux propriétaires du foncier ou du bâti. EDF ne cherche aucunement à rendre "patrimonial », dans le sens culturel, le bâti existant qu'elle considère seulement comme un outil de production d'électricité. Dans une stratégie d'entreprise d'ancrage territorial, la chargée de communication EDF emploie avec grand intérêt le terme «identité » pour désigner leur centrale dans le territoire estuarien. Plus généralement, le responsable du label « Patrimoine architectural du $\mathrm{Xx}^{\mathrm{e}}$ siècle » à la DRAC Pays de la Loire montre qu'il est difficile d'appréhender l'industrie comme patrimoine : "Sur 4 ou 5 sites proposés, il y en a qu'un seul qui est accepté parce que les propriétaires ne veulent pas en entendre parler non plus. Ils veulent être libres de toute action sur leur objet. Même si on leur dit qu'il n'y a aucune servitude. Ils se méfient. »

Enfin, le troisième facteur vient des acteurs du patrimoine - DRAC et Service de l'inventaire qui ne tiennent pas compte de cette centrale dans leurs actions et leurs démarches. Cela vient tout d'abord du fait qu'il n'y ait eu aucune demande du 
propriétaire (EDF). De son côté, le responsable du label « Patrimoine architectural du Xx siècle » à la DRAC Pays de la Loire explique qu'il n'y a pas eu de demande du propriétaire ou d'une personne morale (association ou autre), aucun dossier n'a été instruit pour EDF. Il avance que la raison en est que cet équipement est encore en activité : "C'est pas $d u$ tout à l'ordre du jour car c'est une centrale qui fonctionne actuellement. »

\section{La centrale thermique au regard de la classification patrimoniale}

Le cas de la centrale thermique est révélateur des ambiguïtés et des contradictions de l'approche patrimoniale appliquée à ce type de bien. L'intérêt de la centrale thermique d'un point de vue patrimonial réside dans les interactions entre ses dimensions paysagères et sa valeur d'usage. Cependant, c'est justement le fait qu'elle soit encore en activité et qu'elle ait une architecture qui dénote dans le paysage préservé et protégé de l'estuaire qui lui empêche son entrée dans le monde du patrimoine officiel.

L'analyse des perceptions des acteurs territoriaux montre une conception de plus en plus fragmentée et ambiguë de ce qui lui confère une valeur particulière. Tout d'abord, la centrale permet d'actualiser, de perpétuer un patrimoine, le port de Cordemais. Par ce phénomène de pérennisation, elle tend à s'ancrer sur le territoire, mais aussi dans son histoire. Elle est acceptée comme un élément de l'histoire de la commune. Le maire de Cordemais met en évidence que la centrale "fait partie de son passé et son devenir ». À l'échelle de l'estuaire, la plupart des personnes rencontrées montrent que la centrale thermique fait partie de l'identité de l'estuaire car elle était liée à son histoire et à son évolution. Par exemple, le directeur du GIP Loire estuaire avance que "l'activité économique elle est une composante de l'Estuaire ». L'industrialisation de l'estuaire commence au XVIII ${ }^{\mathrm{e}}$ siècle. Par ailleurs, les aménagements effectués au $\mathrm{Xx}^{\mathrm{e}}$ siècle influencent le paysage et le fonctionnement de l'estuaire. La centrale thermique est représentative de ce siècle. La directrice d'Estuarium évoque le fait qu'elle la fait visiter comme un élément caractéristique des aménagements récents : «Après la centrale EDF, quand on parle avec les scolaires de l'évolution de l'industrie dans l'Estuaire, ça nous arrive souvent de terminer notre exposé ou notre parcours à Cordemais parce qu'elle illustre l'industrie du XXe siècle. »

La deuxième valeur mise largement en avant par tous les acteurs est sa présence dans le paysage. Cette dimension a été abordée lors de l'élaboration de la biennale «Estuaire » de 2009 et le choix du site de Cordemais pour l'installation d'une œuvre pérenne. La chargée de la communication de l'événement raconte ce rapport de la centrale au paysage : "On est sur un terrain très plat et on a cette centrale électrique qui se voit du pont de Cheviré au pont de Saint-Nazaire. Comment on peut raconter cet espace-là alors que la centrale dit elle-même beaucoup de choses: la place de l'homme dans l'estuaire, la place de l'industrie, la technologie... et il y a la Loire devant qui est violente, rapide. Comment on va pouvoir raconter ça? Comment raconter par l'acte artistique la place de l'homme dans l'estuaire, la place de l'industrie ou encore la place de la technologie que la centrale de Cordemais symbolise? »Dans le même sens, le travail d'Estuarium sur le patrimoine estuarien s'est aussi tourné vers le « patrimoine » dit « industriel » de l'estuaire. Cet ethnopôle a voulu montrer la patrimonialité de la centrale thermique de Cordemais ou encore de la raffinerie de Donges dans son rapport à l'environnement. La directrice de l'association explique pourquoi elle utilise le terme «patrimoine» pour désigner la centrale : «Pour nous, ça s'inscrit dans un territoire, c'est reconnu par un certain nombre de personnes comme ayant toute sa place anjourd'hui sur ce territoire. » Elle ajoute que le point de vue choisie par l'association est de mettre valeur «à la fois les marais et l'industrie » dans les expositions et les ouvrages publiés sur le patrimoine estuarien. Le rapport entre les dimensions paysagères, territoriales et architecturales confère à la centrale une valeur hybride.

Un troisième élément caractéristique de la centrale thermique de Cordemais tend à créer les conditions de sa définition patrimoniale : sa valeur d'usage symbolisée dans son architecture imposante. La plupart des interlocuteurs évoque l'imaginaire autour de l'usage de cette centrale incarné par l'architecture intrigante faite de fer et d'acier. L'imbrication entre matériel et immatériel est très intéressante dans ce cas. Pour les personnes rencontrées, le fonctionnement de la centrale lié à sa silhouette architecturale attire les gens et attise leur curiosité. L'architecture 
démesurée de cette centrale interroge sur son fonctionnement. Le maire de Cordemais explique en quoi l'usage est l'un des éléments les plus importants de la centrale : "La plupart des gens la considère comme du patrimoine parce que c'est en activité. C'est à dire que si vous l'arrêter, je ne suis pas sur qu'ils considèreront ça comme un patrimoine. Moi personnellement, je ne le verrais pas comme un patrimoine. " Il va plus loin dans sa réflexion : «Moi je pense que ce qui est intéressant dans une centrale c'est son activité. Et puis il y a un mystère de la machinerie. Après vous avez qu'une boîte, une boîte qui n'a plus d'âme parce qu'il n'y a plus personne dedans. » Cette valeur d'usage apparaît comme essentielle mais aussi destructrice de patrimonialité. L'obstacle apparent issu de l'activité vient de la difficulté du lien entre activité et ouverture au public. Les règles à suivre sont assez strictes ainsi que les procédures pour entrer sur le site. Les consignes Vigipirate viennent s'ajouter aux règles internes déjà strictes. La visite n'est nullement l'objet premier du travail des agents EDF ou des gestionnaires du site. La production d'énergie passe avant la visite. Face à ce problème, l'évènementiel et la visite ponctuelle reste les deux méthodes les plus adaptées qui permettent l'appropriation des habitants.

\section{Conclusion}

À Cordemais, les conditions de production d'énergie sont liées à l'imbrication de nombreuses caractéristiques. Cette hybridité patrimoniale est mise à mal par l'approche moderne du patrimoine pris dans le double processus d'extension et de catégorisation. D'un côté, la société occidentale «patrimonialise » des biens matériels puis immatériels, des biens anciens puis de plus en plus récents, des lieux à caractère unique puis des territoires de plus en plus banalisés. De l'autre elle les classifie. Dans ce cadre, la valeur hybride de la centrale thermique pose question : est-ce un patrimoine matériel ou immatériel? S'il est matériel, est-ce un patrimoine architectural du $\mathrm{xx}^{\mathrm{e}}$ siècle, un patrimoine industriel, un patrimoine technique, un patrimoine technologique? S'il est immatériel, est-il symbolique, culturel, imaginaire? La centrale thermique est tout cela à la fois et que cette caractéristique atypique la rend difficile à appréhender avec les outils et méthodes traditionnelles du patrimoine.
Pour autant, peut-on et doit-on la questionner comme tel? La continuité de son usage reste le point central du problème. L'ensemble des discours recueillis permettent de répondre positivement. Cependant, la valeur d'usage de la centrale thermique tend à aller à l'opposée de tout questionnement patrimonial. À l'inverse, si l'activité cesse, il apparait clairement que l'intérêt de cet équipement disparaitra avec elle. EDF mène d'ailleurs une politique dans ce sens en détruisant ses centrales hors d'usage pour rendre le terrain aux collectivités territoriales. Sans valeur d'art ou technique particulière, la valeur particulière que la population et que les acteurs territoriaux lui accordent semble éphémère. Pour l'instant, cet objet n'a pas besoin d'être conservé ou converti. Sa désignation comme objet «identifiant » ne pose donc pas de problème de coûts. Il doit être en état pour fonctionner. La question patrimoniale surgira lors de l'arrêt de la production. Pour l'instant, personne ne souhaite sa «patrimonialisation » officielle.

\section{Remerciements}

Cette recherche a été soutenue par le FEDER au titre du Plan Loire Grandeur Nature et par l'Établissement Public Loire dans la cadre de la thèse de Clément Colin.

\section{Bibliographie}

Amougou E., 2011. Sciences sociales et patrimoines, Paris, L'Harmattan, $169 \mathrm{p}$.

Andrieux J.-Y., 1992. Le patrimoine industriel, Paris, PUF, coll. "Que sais-je?», 127 p.

Bianchini A., 2008. Le tourisme industriel est en vogue, Ministère des Affaires Etrangères et Européennes, Direction de la communication et de l'information, $n^{\circ} 33$, [En ligne] : [http://www.ambafrance-kh.org].

Bourdin A., 1984. Le patrimoine réinventé, Paris, PUF, 239 p.

Brubaker R., 2001. Au-delà de l'« identité », Actes de la recherche en sciences sociales, $\mathrm{n}^{\circ} 139$, p. 66-85.

Choay F., 1992. L'allégorie du patrimoine, Paris, Le Seuil, 270 p.

Davallon J., 2006, Le don du patrimoine, Paris, Lavoisier, $222 \mathrm{p}$.

Di MÉo G., 1994. Patrimoine et territoire, une parenté conceptuelle, Espaces et Sociétés, n ${ }^{\circ}$ 8, p. 15-34.

Di Méo G., 1998. Géographie sociale et territoires, Paris, Nathan Université, 320 p. 
Edelblutte S., 2008. Paysages et territoires du patrimoine industriel au Royaume-Uni, Revue Géographique de l'Est, vol. 48, n 1-2, [En ligne] : [http://rge.revues.org/648].

EDF, 2009. EDF, acteur du développement de la culture scientifique et technique, Dossier de Presse, EDF, 18 p.

EDF, 2010. Déconstruction de la cheminée de la tranche 1 de la Centrale de Cordemais, Dossier de Presse, EDF, 8 p.

Gravari-Barbas M. (dir.), 2005. Habiter le patrimoine : enjeux, approches, vécus, Rennes, PUR, 618 p.

Gravari-Barbas M., Veschambre V., 2003. Patrimoine : derrière l'idée de consensus, des enjeux d'appropriation de l'espace et des conflits, dans Melé P. (dir.), Conflits et territoires, Tours, Presses universitaires François-Rabelais, p. 67-82.

Guillaume J., 2009. L'avenir du pôle industriel et portuaire de l'estuaire de la Loire, in Desprès L. (dir). L'Estuaire de la Loire, un territoire en développement durable?, Rennes, PUR, p. 171-194.

Halgand M.-P., Guillaume J., 2007. Basse Loire, Une histoire industrielle, Nantes, Ed Mémo, Carnets d'Usines, 127 p.

Jeudy H.-P, 2008. La Machine Patrimoniale, Éditions Circé, $121 \mathrm{p}$.

Kerouanton J.-L., Lemaître Y., 1996. Cordemais en Estuaire Loire Atlantique, Itinéraires du Patrimoine, Ministère de la Culture et de la Communication, 18 p.
LAmy Y., 1993. Du monument au patrimoine : Matériaux pour l'histoire politique d'une protection, Genèses, $n^{\circ} 11$, p. $50-81$.

Lemaître Y., Lemerle E., 2004. Les « petits ports » de la BasseLoire ou la «face cachée de l'estuaire », Aestuaria, Cultures et développement durable, $\mathrm{n}^{\circ}$ 5, Estuarium, Cordemais, p. 285-314.

Marcon A., Preuilh P., Ksouri S., 2000. Tourisme de découverte économique et visites d'entreprises, Paris, La Documentation française, $164 \mathrm{p}$.

Morice J.-R., 2006. La visite d'entreprise en Europe, Un champ à explorer, Espaces, Edition tourisme et loisir, n 92 , p. $10-15$.

Poulot D. (dir.), 1998. Patrimoine et Modernité, Paris, L'Harmattan, $311 \mathrm{p}$.

Riegl A., 1903. Le culte moderne des monuments, Paris, L'Harmattan, $124 \mathrm{p}$.

Veschambre V., 2007. Patrimoine : un objet révélateur des évolutions de la géographie et de sa place dans les sciences sociales, Annales de géographie, 2007/4, n 656, p. 361-381.

Veschambre V., 2008. Traces et Mémoires urbaines, Enjeux sociaux de la patrimonialisation et de la démolition, Rennes, PUR, 310 p. 